\title{
Sekuritisasi Data Sensor Pada Aplikasi Internet of Things (IoT) Dengan Menggunakan Blockchain Ethereum Di Jaringan Testnet
}

\author{
Joni Fat ${ }^{1}$, Henry Candra ${ }^{2}$, William ${ }^{3}$
}

\begin{abstract}
Internet of Things (IoT) and blockchain are two technologies which are pioneer nowadays and in the future. IoT become pioneer because our goverment sets Industrial Revolution 4.0 which signifies the IoT technology. For blockchain, it is a technology which has a significant development. These two technologies have a good future. The innovation in this design is combining these two technologies. IoT has a problem in securitization, but blockchain has an advantage in security. So, by combining these technologies, the problem could be resolved. This design used microcontroller ESP32. It will collect three data sets, i.e. altitude, pressure and temperature. The data will be sent through Internet to Ethereum blockchain network in Ropsten TestNet. The Data will store in state variables. Twenty data have been sent and tested. The data has been verified to be stored in Ethereum Virtual Machine because the data could be verified through etherscan.io. So, the conclusion is this design could prove that blockchain could be used to data securitization in IoT.
\end{abstract}

KEYWORD: blockchain, ethereum, ESP32, IoT, securitization

ABSTRAK: Internet of Things (IoT) dan blockchain merupakan dua teknologi yang akan menjadi pioneer saat ini dan di masa mendatang. Pioneer saat ini karena pemerintah menetapkan Revolusi Industri 4.0 yang salah satu titik beratnya adalah IoT. Untuk blockchain, saat ini merupakan teknologi yang mengalami perkembangan signifikan. Kedua teknologi ini menjanjikan. Salah satu hal yang dapat menjadi inovasi adalah menggabungkan keduanya. IoT memiliki permasalahan pada sekuriti, sedangkan blockchain memiliki keunggulan dalam hal sekuriti. Dengan demikian, menggunakan teknologi blockchain untuk aplikasi IoT akan menjadi solusi. Alat yang dirancang menggunakan mikrokontroler ESP32. Alat ini akan mengakuisis data dari tiga buah sensor, yaitu sensor ketinggian, tekanan dan suhu. Data-data ini dikirim melalui Internet ke jaringan blockchain Ethereum di TestNet Ropsten. Data tersebut akan disimpan di state variables. Dua puluh data dikirim dan berhasil disimpan di Ethereum Virtual Machine. Ini terbukti dengan setiap data yang dikirim dapat divalidasi dan terekam di etherscan.io. Dengan demikian, perancangan alat ini berhasil membuktikan bahwa IoT dapat menggunakan blockchain untuk sekuritisasi data.

KATA KUNCI: blockchain, ethereum, ESP32, IoT, sekuritisasi

\section{PENDAHULUAN}

$I$ nternet of Things (IoT) merupakan salah satu fokus dalam Revolusi Industri 4.0. IoT adalah gelombang perubahan berikutnya setelah era Internet. Diperkirakan akan ada 46 milyar peralatan yang terhubung ke Internet pada tahun 2021 [1]. Peralatan yang terhubung ke Internet ini, mulai dari Body Area Network (BAN), berupa jaringan dengan peralatan di sekitar tubuh, hingga jangkauan yang sangat luas. Ini berarti akan banyak data sensitif yang akan terekspos. Hingga saat ini, isu sekuritisasi data ini masih menjadi pokok permasalahan, karena berdasarkan hasil penelitian yang dipublikasi oleh HP, 70\% komunikasi IoT tidak terenkripsi [2]. Dengan bertambahnya popularitas blockchain, timbul ide untuk memadukan blockchain dan IoT.

Blockchain memiliki keunggulan dari sisi sekuritisasi. Enkripsi dalam blockchain memberikan harapan bahwa sekuritisasi komunikasi mau pun data IoT dapat dilakukan dengan baik. Walau pun hal tersebut masih diiringi dengan beberapa kekurangan dari teknologi Blockchain. Teknologi Blockchain mengggunakan kritografi secara masif [3]. Dengan demikian, blockchain dapat digunakan untuk menjamin transaksi dalam jaringan yang memiliki tingkat keamanan yang rendah seperti Internet. Setiap blok dalam blockchain diidentifikasi dengan sebuah kode hash dan nonce. Kedua kode ini bersifat unik. Setiap blok saling berkaitan dengan blok sebelumnya. Bila sebuah blok diubah, maka keseluruhan rantai bloknya harus diubah juga. Hal ini mengakibatkan, pengubahan blok sangat sulit dilakukan dengan daya komputasi saat ini. Selain itu, teknologi blockchain juga memanfaatkan metode konsensus [3]. Ini berarti setiap transaksi dalam blockchain harus mencapai nilai konsensus tertentu agar tervalidasi dalam jaringannya. Blok juga terduplikasi dalam setiap server dalam jaringan server tersebut. Dengan demikian, dapat dipahami bahwa untuk mengubah transaksi dalam blockchain secara tidak sah akan sangat sulit dilakukan. Oleh karena itu, blockchain memiliki sekuritisasi transaksi yang mustahil untuk diubah secara tidak sah dengan daya komputasi saat ini. Danzi [4] menunjukkan upaya untuk membangun konsep arsitektur IoT dan blockchain untuk menyelesaikan persoalan sekuritisasi dalam metode pembayaran mikro. Danzi membangun model penyelesaian tersebut. Danzi menggunakan mikrokontroler sebagai peralatan IoT terhubung dengan jaringan blockchain. Interaksi antara mikrokontroler dan jaringan blockcahin ini bersifat irreversible dan tanpa perlu mutual trust.

Dengan demikian, dapat disimpulkan bahwa mikrokontroler merupakan bagian utama, karena semua proses dimulai dari mikrokontroler ini. Sensor, aktuator, dan peralatan lain terhubung dengan mikrokontroler. Oleh mikrokontroler data diakuisisi atau diproses dan diteruskan ke modul jaringan yang juga terhubung dengan mikrokontroler. Dapat dikatakan bahwa semua proses IoT dimulai di mikrokontroler. Oleh sebab itu, proses yang dalam sekuritisasi adalah proses sekuritisasi dalam pemrosesan oleh mikrokontoler ini. Jadi, untuk dapat memastikan bahwa blockchain dapat diaplikasikan pada peralatan IoT, pada perancangan ini akan menggunakan mikrokontroler untuk menunjukkan bahwa signing dapat dilakukan [5]. Penggunaan mikrokontroler ini memenuhi tujuan pembuktian bahwa blockchain dan IoT dapat dipadukan.

Beberapa penelitian yang telah dilakukan terkait dengan IoT dan blockchain adalah sebagai berikut: 
- U. Guin, P. Cui dan A. Skjellum [6] membuat model sistem menggabungkan IoT dan blockchain dalam rangka menangani metode pembayaran mikro. Dalam penelitian ini, para peneliti mengusulkan arsitektur dan model yang sesuai.

- A. Dorri, et. al. [7] membuat studi kasus dan simulasi perihal pentingnya sekuritisasi IoT untuk aplikasi smart home. Para peneliti menggunakan blockchain untuk sekuritisasi dan privatisasi data.

- S. F. T. O. Mendonca, J. F. S. Junior dan F. M. R. Alencar [8] menjelaskan tentang tantangan yang dihadapi teknologi IoT berbasis blockchain. Systematic Mapping yang digunakan peneliti ini menemukan adanya threads of validity.

- J. Kogure, et. al. [9] memberikan gambaran tentang teknologi blockchain beserta aplikasi contohnya. Para peneliti juga mengusulkan alur dalam proses cross-border transactions.

- A. Dorri, et. al. [10] mengusulkan Lightweight Scalable Blockchain (LSB) untuk sekuritisasi dan privatisasi data IoT. Ini merupakan model teknologi yang dioptimasi untuk IoT.

\section{DIAGRAM BLOK}

Modul klien adalah berupa mikrokontroler ESP32 yang memiliki kemampuan untuk melakukan signing terhadap transaksi blockchain. Agar dapat berinteraksi ke jaringan Ethereum, perlu adanya node Remote Procedure Call (RPC). Dalam sistem ini, akan menggunakan Infura. Infura akan menjembatani data dari modul klien ke jaringan Ethereum. Data transaksi tersebut kemudian akan diverifikasi oleh Ethereum Virtual Machine (EVM) yang terdapat di dalam jaringan TestNet. Hasil verifikasi kemudian dapat dimonitor melalui situs etherscan.io.
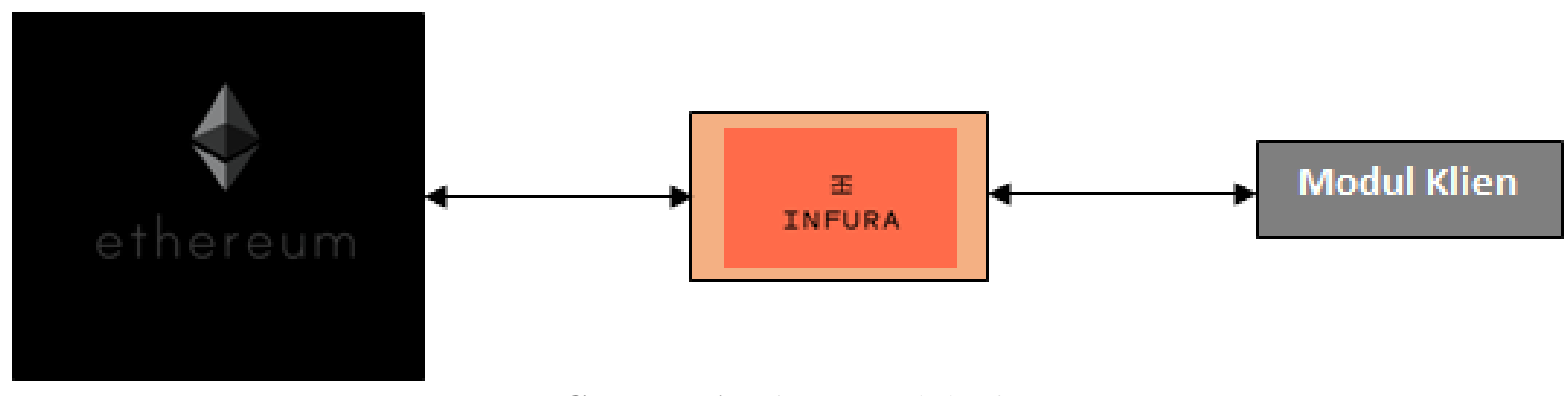

- Gambar 1. Diagram Blok Sistem

\section{INPUT, OUTPUT DAN PROSES}

Sistem yang dirancang dalam bentuk input, output dan proses dapat dilihat pada Tabel 1 berikut. Input dalam sistem ini dibagi menjadi data jumlah sensor (validasi), data sensor (data-data), dan time_stamp. Proses dalam sistem ini terdiri dari proses akuisisi data, timer untuk penanda waktu pengiriman data, proses signing dan proses validasi data. Output sistem adalah data yang tervalidasi dalam blok. Ini diperiksa melalui etherscan.io.

- Tabel 1. Input, Output dan Proses

\begin{tabular}{|c|c|c|}
\hline Input & Proses & Output \\
\hline Jumlah Sensor & Akuisisi & \multirow{2}{*}{ Data tercatat dalam blok } \\
\hline Data-Data Sensor & Timer & \\
\hline Time_Stamp & Signing & \\
\hline & Validasi & \\
\hline
\end{tabular}

\section{PARAMETER NON-FUNCTIONAL}

Berikut adalah parameter-parameter keberhasilan non-functional terhadap sistem:

- Penyusunan Smart Contract yang tepat,

- Ketersedian ETH karena setiap transaksi pencatatan ke jaringan Ethereum memerlukan sejumlah nilai ETH tertentu, 
- Keandalan koneksi Internet.

\section{FORMAT DATA}

Sesuai dengan namanya, blockchain merupakan sekumpulan blok yang saling berkaitan membentuk sebuah rantai atau linked list. Setiap blok terdiri dari header dan body. Dalam header dan body terdapat sekumpulan fields. Banyak dan isi dari fields adalah sesuai dengan spesifikasi protokol masing-masing blockchain.

Header biasanya terdiri dari kode Hash dan nonce yang merupakan pointer untuk blok berikutnya dan kode unik. Kedua kode tersebut merupakan solusi PoWs. Selain itu, juga terdapat data lain seperti waktu pembuatan dan roots dari pohon Merkle. Body terdiri dari data transaksi yang memerlukan verifikasi. Body biasanya memiliki ukuran maksimum.

\section{PROTOKOL BLOCKCHAIN}

Blockchain menekankan Proof of Works (PoWs) sebagai cara untuk melakukan validasi. Validator menggunakan metode konsensus dan mempercayai blockchain yang paling panjang dalam jaringan [4]. Blockchain pada prinsipnya terdiri dari sekumpulan blok yang disimpan dalam bentuk copy oleh nodes dalam jaringan blockchain. Apabila ada penambahan blok baru, maka blok tersebut akan di-update keseluruh nodes dalam jaringan. Agar dapat ditambahkan, validasi dilakukan. Inilah yang dinamakan dengan PoWs.

Algoritma PoWs dijalankan secara lokal oleh validator atau node. Dalam hal jaringan Ethereum, dilakukan oleh EVM. Tugas dari algoritma ini adalah untuk mencari solusi terhadap enkripsi kriptografi dalam blok tersebut. Algoritma PoWs memiliki tujuan utama untuk mencegah penambahan blok baru oleh pihak lain dan menjamin agar setiap nodes mendapatkan copy block yang konsisten [11].

\section{METODOLOGI}

Hal pertama yang dilakukan dalam penelitian ini adalah melakukan instalasi wallet Ethereum dengan menggunakan Metamask. Ini akan menjadi bagian pertama dalam melakukan interaksi dengan jaringan blockchain Ethereum. Dalam interaksi, diperlukan sejumlah nilai Ether sebagai biaya untuk proses. Oleh karena itu, setelah wallet terinstalasi, perlu meminta sejumlah nilai Ether ke jaringan TestNet. Jaringan TestNet yang digunakan adalah Ropsten. Selanjutnya, mikrokontroler dan program untuk melakukan signing serta smart contract perlu dibuat. Pengujian merupakan langkah berikutnya. Bila pengujian gagal, maka perlu diulangi proses dari meminta Ether. Bila berhasil, akan dapat diperiksa melalui etherscan.io.

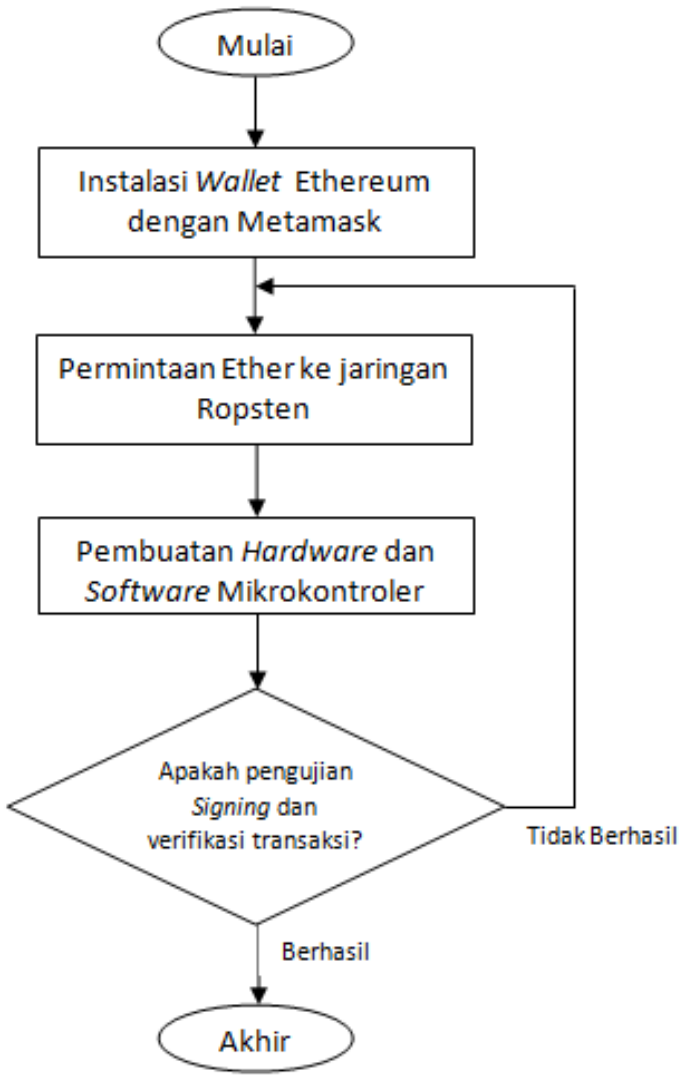

- Gambar 2. Metodologi 


\section{HASIL RANCANGAN}

Rancangan berupa modul klien yang terdiri dari mikrokontroler ESP32 dan tiga buah sensor. Ketiga buah sensor tersebut adalah berupa sensor ketinggian, tekanan dan suhu. Sensor-sensor ini merupakan sensor yang didukung oleh mikrokontroler ESP32. Pemrograman di ESP32 menggunakan bahasa Phyton dengan library dari Zerinth. Oleh karena library ini mendukung akses ke blockchain Ethereum. Untuk program di Ethereum Virtual Machine (EVM) digunakan bahasa Solidity yang merupakan bahasa untuk memprogram smart contract Ethereum. Smart contract ini di-deploy dengan menggunakan remix.ethereum.org. Hasilnya adalah smart contract yang live di jaringan TestNet Ropsten dengan alamat 0x9Ad8Ee5E185455D6E7205bF63cE3808EDa44A2Ff. Private key-nya adalah 0x33627590e0b8751ddabec6d0278c1e5f7da1821c9eb2a7b06f5d0edb3ec579ae. Wallet yang digunakan memiliki alamat 0x81b7e08f65bdf5648606c89998a9cc8164397647.

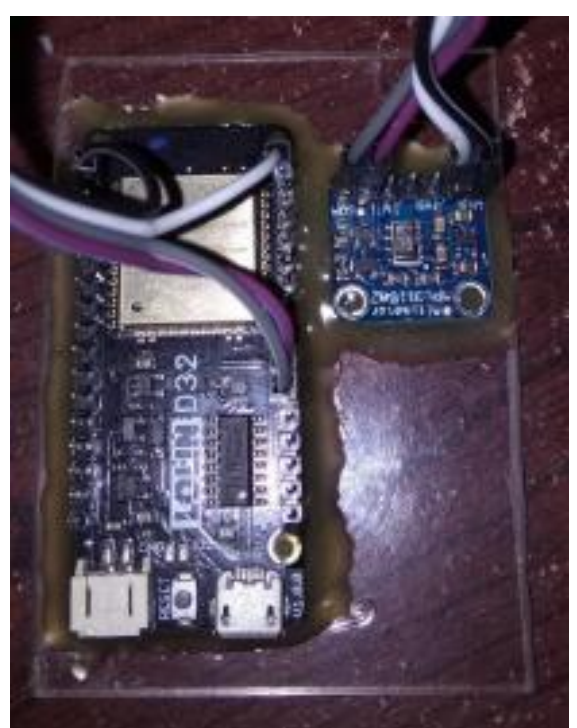

- Gambar 3. Hasil Rancangan

\section{PENGIRIMAN DATA}

Pengiriman data dilakukan setiap 30 detik karena ini adalah waktu minimal yang diperlukan oleh EVM untuk memvalidasi data sebelumnya. Waktu 30 detik ini didapatkan dengan melakukan pengujian. Setelah pengujian berkali-kali dengan melakukan penambahan waktu per detik, didapatkan bahwa hanya setelah delay 30 detik, data berikutnya dapat diterima dan divalidasi oleh EVM. Seperti telah disinggung sebelumnya, data yang dikirim adalah tiga jenis data yang diakuisisi dari sensor ketinggian, tekanan dan suhu. Gambar 4 berikut memperlihatkan prosedur pengiriman data dari modul klien ke EVM di jaringan TestNet Ropsten. Pertamatama, data dibaca oleh setiap sensor, setelah itu data dikirimkan dengan bantuan infura.io ke EVM. Dari gambar ini terlihat, data yang dikirimkan dan biaya yang perlu dibayarkan dalam hitungan gas.

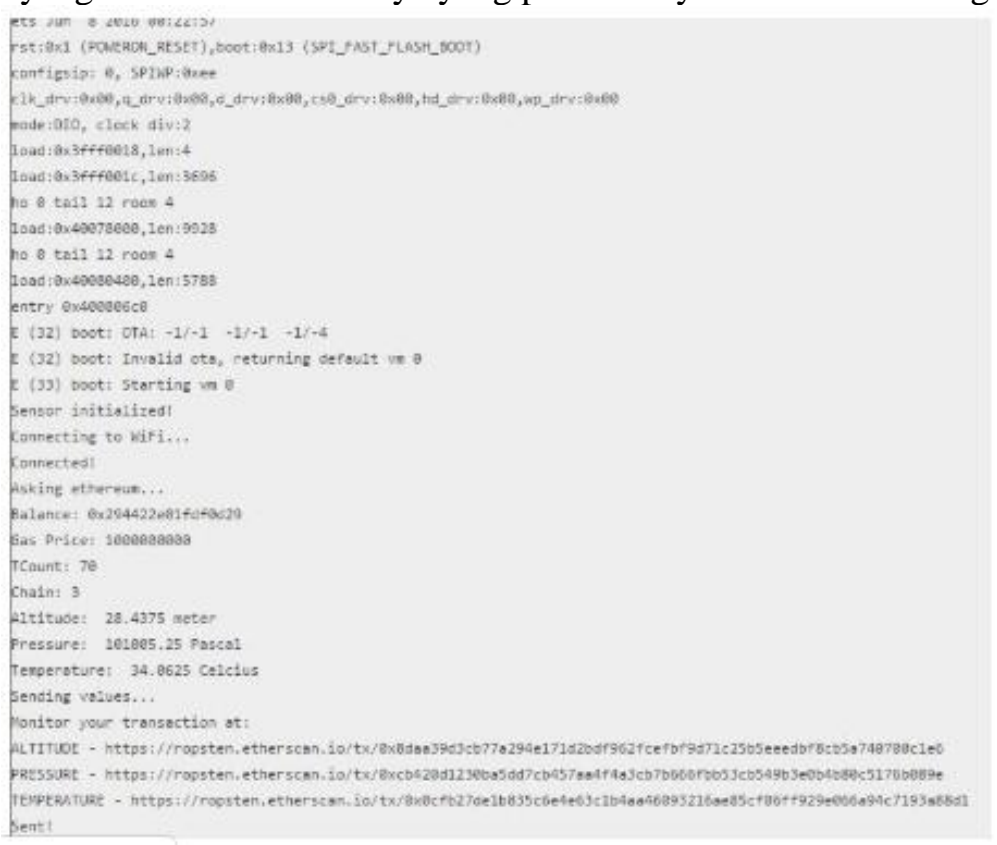

- Gambar 4. Pengiriman Data dari Modul Klien ke EVM Jaringan TestNet Ropsten 


\section{KETINGGIAN}

Gambar 5 memperlihatkan bukti pengiriman data ketinggian ke jaringan TestNet Ropsten. Pada gambar ini terlihat status pengiriman telah berhasil, besar biaya yang dibayarkan dalam memproses transaksi tersebut (dalam gas), dan juga alamat pengirim serta alamat smart contract sebagai lokasi penyimpanan data. Gambar 6 memperlihatkan 20 data ketinggian yang dikirimkan sebagai pengujian pengiriman data ketinggian. Setiap data yang dikirimkan disertai dengan bukti keberhasilan pengiriman data yang dapat diperiksa di etherscan.io. Setiap link data akan memperlihatkan status pengiriman, jumlah validasi, besar biaya transaksi dan data yang direkam.

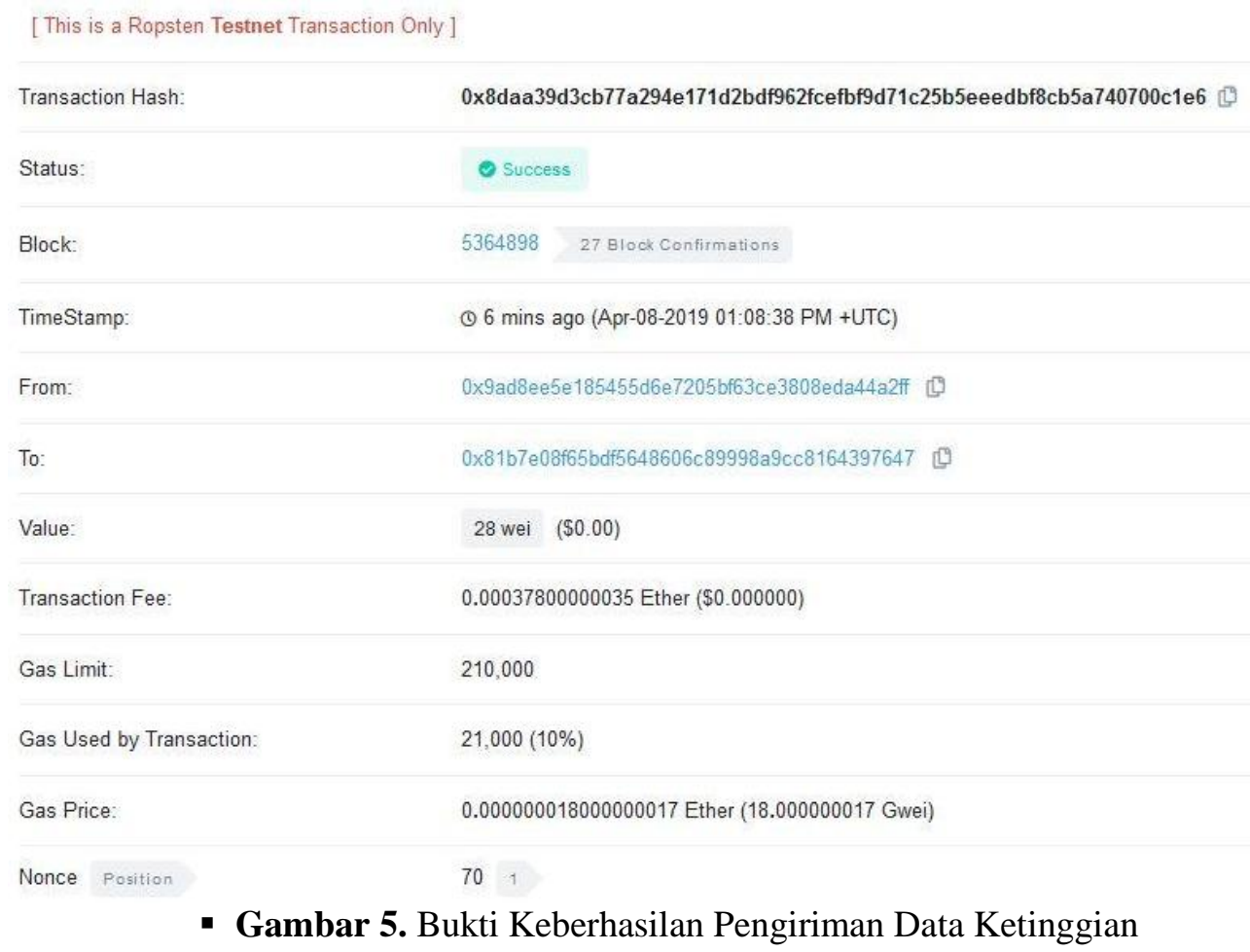

\begin{tabular}{|c|c|}
\hline Value & Etherscan \\
\hline $30.5 \mathrm{~m}$ & https://ropsten.etherscan.io/tx/0xf2cbc27bc6c28d5c2ec903350867e21e98a6e29efe1f48c19d053c8d7bf64ed0 \\
\hline $34.375 \mathrm{~m}$ & https://ropsten.etherscan.io/tx/0x6070507b2afb51b5a21915bb3fbf86b22d241969cd2f5fcc $9729507 f 2 a a 51304$ \\
\hline $36.625 \mathrm{~m}$ & https:/ropsten.etherscan.io/tx/0xb1bf2317807f3ac246c84acb0d836519606f0b110a6163af64085e99b6726b29 \\
\hline $36.375 \mathrm{~m}$ & https://ropsten.etherscan.io/tx/0x1044fc4d2ee2aeacf6ce8ef770b8ac543ef26393afac491caf4235a128ccb6b3 \\
\hline $37.4375 \mathrm{~m}$ & https://ropsten etherscan.io/tx/0x8d53df99110648427b0fc447b861fbd81a299ad1215d3ba2667018e200f0bc69 \\
\hline $37 \mathrm{~m}$ & https://ropsten.etherscan.io/tx/0x68c9356a3ad67d0b5c6ef2e2523e69fc191e16a7a351b33fd8519ba8b6c93772 \\
\hline $36.125 \mathrm{~m}$ & https $/ /$ ropsten.etherscan.io/tx/0x $76462866 \mathrm{f} 736 \mathrm{~d} 2790 \mathrm{cb} 79860 \mathrm{bc} 421 \mathrm{a} 3 \mathrm{e} 4 \mathrm{dc} 08746988941915 \mathrm{c} 61 \mathrm{~d} 48 \mathrm{a} 7969238 \mathrm{a}$ \\
\hline $33.6875 \mathrm{~m}$ & https:/ropsten.etherscan.io/tx/0x23bd6ce4470979b0191dafe37d1dlf2583367be4d71a691290f2eedfcb461390 \\
\hline $35.375 \mathrm{~m}$ & https $/ /$ ropsten .etherscan.io/tx/0xba0c0d76bealb9c9c017777b43c38acb097ba91f8d6568171dc40b7d7f363658 \\
\hline $29.375 \mathrm{~m}$ & https://ropsten etherscan.io/tx/0x01ebba317bebeb3d131b8310fb644c3953cb7ad6ae3647baf570621e8cc240b8 \\
\hline $37.8125 \mathrm{~m}$ & https $/ /$ ropsten .etherscan.io/tx/0x95ce985da900859c2d885d4b20872c4e2bfb01da328a3dac27f7b13d166926c9 \\
\hline $37.5625 \mathrm{~m}$ & https://ropsten.etherscan.io/tx/0xaa3b194a2e3a4dcdc0fdf068d88c36cc48e71ec14cfd61f6902936e35328fb99 \\
\hline $35.5 \mathrm{~m}$ & https://ropsten.etherscan.io/tx/0xe4alf1d5a425b82bf9c759aa2766a33678f2c6ed31b6d658276dc33bb9f872f 48 \\
\hline $34.1875 \mathrm{~m}$ & https $/ /$ ropsten.etherscan.io/tx/0x $24 b 701672811$ f0866d21fefcb3bc8cd4fe 8 e682f5582146144597d2f4e90583c \\
\hline $32.4375 \mathrm{~m}$ & https $/ /$ ropsten.etherscan.io/tx/0x1e469d22187aec9a0a2625e7b21d0094652df143beb423fec $48 \mathrm{~d} 8 \mathrm{ca} 400 \mathrm{f} 01 \mathrm{f5e}$ \\
\hline $32.8125 \mathrm{~m}$ & https://ropsten.etherscan.io/tx/0x84ba0a2873c3647ab8e2f61289e06c465e653045c45558def3cb7a344e9a1e1d \\
\hline $32.8125 \mathrm{~m}$ & https $/ /$ ropsten etherscan.io/tx/0x2e47feefb6d27bd1671955bacce2de64d6524770f5c $43 \mathrm{~d} 04 \mathrm{f} 815 \mathrm{af} 9 \mathrm{f5} 39 \mathrm{f} 0442$ \\
\hline $33.875 \mathrm{~m}$ & https://ropsten.etherscan.io/tx/0xe94b3e4b3cb106befe $8689 \mathrm{~b} 5 \mathrm{eb} 2 \mathrm{c} 47343906786 \mathrm{efe} 792069 \mathrm{ee} 608 \mathrm{e} 23 \mathrm{a} 50 \mathrm{eaa} 37$ \\
\hline $29.25 \mathrm{~m}$ & https://ropsten.etherscan.io/tx/0x98e55ffe $955555014 \mathrm{f} 773 \mathrm{ef} 214 \mathrm{f} 59717 \mathrm{a} 7 \mathrm{dc} 072 \mathrm{~d} 5 \mathrm{bd} 7 \mathrm{dc} 51 \mathrm{~b} 85 \mathrm{da} 91 \mathrm{a} 67 \mathrm{f} 3 \mathrm{fcc} 7$ \\
\hline $34.0625 \mathrm{~m}$ & https://ropsten.etherscan.io/tx/0x5db0223ee9dbd862f2ee8b7eccf13e53352f253ef84cf5738c097632e 1802829 \\
\hline
\end{tabular}

- Gambar 6. Data Pengujian Sensor Ketinggian dan Hasil Validasi

\section{TEKANAN}

Data sensor yang diuji berikutnya adalah data sensor tekanan. Sensor tekanan dipilih karena sensor ini sering digunakan untuk berbagai peralatan IoT. Gambar 7 menunjukkan bukti pengiriman data sensor tekanan yang berhasil dilakukan. Berhasil di sini dimaksudkan bahwa data telah terekam di EVM. Ini terbukti data dapat divalidasi kembali. Pada gambar ini ada satu variabel yang dinamakan Gas Limit ini merupakan nilai maksimum yang diinginkan dalam transaksi perekaman data ke EVM. Di gambar ini juga terlihat bahwa biaya transaksi hanya sebesar 10\% dari batas maksimum yang ditetapkan. Gambar 8 memperlihatkan 20 data dari 
sensor tekanan yang digunakan untuk pengujian. Setiap data ini disertai link di situs etherscan.io. Ini berarti setiap data ini dapat dilihat status keberhasilannya dan juga dapat diperiksa kembali.

\begin{tabular}{|c|c|}
\hline Transaction Hash: & 0xcb420d1230ba5dd7cb457aa4f4a3cb7b666fbb53cb549b3e0b4b80c5176b089e (t) \\
\hline Status: & - Success \\
\hline Block: & 536490325 Block Confirmations \\
\hline TimeStamp: & (1) 6 mins ago (Apr-08-2019 01:09:41 PM +UTC) \\
\hline From: & 0x9ad8ee5e185455d6e7205bf63ce3808eda44a2ff [t] \\
\hline To: & Ox81b7e08f65bdf5648606c89998a9cc8164397647 [0] \\
\hline Value: & 0.000000000000101005 Ether $\quad(\$ 0.00)$ \\
\hline Transaction Fee: & 0.00037800000035 Ether $(\$ 0.000000)$ \\
\hline Gas Limit: & 210,000 \\
\hline Gas Used by Transaction: & $21,000(10 \%)$ \\
\hline Gas Price: & 0.000000018000000017 Ether $(18.000000017$ Gwei) \\
\hline Nonce Position & 7. Bukti Pengiriman Data Sensor Tekanan \\
\hline
\end{tabular}

\begin{tabular}{|c|c|}
\hline Value & Etherscan \\
\hline $100923 \mathrm{~Pa}$ & https://ropsten.etherscan.io/tx/0x4f6d7135a576424b95a437254bee344ffc5c8d6f7d7b82a327466e4b986c5dbd \\
\hline $100940.5 \mathrm{~Pa}$ & https://ropsten.etherscan.io/tx/0xa022ad3b30a6a96b8078c2f8acb4dd7a3ebebc1b909385f024dfb4e1bc3707d9 \\
\hline $100938.25 \mathrm{~Pa}$ & https://ropsten etherscan.io/tx/0xf11149d5d56854e67ce2d5fde4a8bc4ecd367ed471529b96c059170bcb416136 \\
\hline $100901.75 \mathrm{~Pa}$ & https://ropsten.etherscan.io/tx/0x333bf389d9aflfa3a56b111feb738937bec41c1791d6d66c2192adebc4410b9a \\
\hline $100895.25 \mathrm{~Pa}$ & https $/ /$ ropsten.etherscan.io/tx/0x6b4c9be2c30e224b2278700dcb7982bfadceafc 7 ddf5 $47871 \mathrm{f} 708 \mathrm{f} 37 \mathrm{c} 01655 \mathrm{a} 7$ \\
\hline $100924.5 \mathrm{~Pa}$ & https://ropsten.etherscan.io/tx/0x684480cd1555b124e94a777b1ca4a7714eldc22b61bf21180d00193d417127e5 \\
\hline $100874.5 \mathrm{~Pa}$ & https:/ropsten.etherscan.io/tx/0xae2a204207flbfa5b9374f8daf47f56efedaa68711d4ef844b73aa177e227c2b \\
\hline $100854.75 \mathrm{~Pa}$ & https://ropsten.etherscan.io/tx/0xd0f34e9e7c92fe1966ae8207016255f4c06b8bc1280aa38ea40019a0b8211109 \\
\hline $100926.75 \mathrm{~Pa}$ & https://ropsten etherscan.io/tx/0x09d01a85337c06fbbc0c200bf08429126950cdb4494d9676b8b8432966afe515 \\
\hline $100927.75 \mathrm{~Pa}$ & https $/ /$ ropsten.etherscan.io/tx/0x65c4a4b19d2d07ae171088a64b7e9c2f9c49672f3a2ba91f7051c17cd56c009a \\
\hline $100926.5 \mathrm{~Pa}$ & https://ropsten etherscan.io/tx/0xb5609164eade1795e84efb38aa7fdfbfe5c1df8a8df07ff06f39074219dlae75 \\
\hline $100903.5 \mathrm{~Pa}$ & https://ropsten.etherscan.io/tx/0xb61c2d4ccd047e9054a18386f0ef2250567f758f61ee319939fc $90 \mathrm{e} 42 \mathrm{df} 215 \mathrm{e} 0$ \\
\hline $100914.75 \mathrm{~Pa}$ & https://ropsten etherscan.io/tx/0xcd33e3c8d8492e1931a3bae7682b5e1c6d803ca8745786403061bd5ceab7b372 \\
\hline $100881.75 \mathrm{~Pa}$ & https://ropsten.etherscan.io/tx/0x61f5c8f1431bf9618c5fdf9da26df341ab3fb97429cb98bb7ffdeba2e693e0d7 \\
\hline $100976.5 \mathrm{~Pa}$ & https $/ /$ ropsten.etherscan.io/tx/0xefa5e3c60falc9751 ca62c9bfb92ca2188754cf6837edeae3e97ed23a7e323a0 \\
\hline $100983 \mathrm{~Pa}$ & https://ropsten.etherscan.io/tx/0x7c969efe4ca9d547dce06426bb1d8660a8f89bb0bdlcfd5156321d9a9592c792 \\
\hline $100931.5 \mathrm{~Pa}$ & https://ropsten.etherscan.io/tx/0xfb00adae46fd13dfce1274134e7d5a0fe5f7f593dd07af2cfd272d8fbb4f1 150 \\
\hline $100913.25 \mathrm{~Pa}$ & https://ropsten.etherscan.io/tx/0x086383386ce7449160100559ceflfd $85 \mathrm{~b} 5 \mathrm{f} 43 \mathrm{ae} 0 \mathrm{f} 74067 \mathrm{~b} 22986 \mathrm{~b} 2988 \mathrm{~d} 0 \mathrm{ed} 774$ \\
\hline $100969.75 \mathrm{~Pa}$ & https://ropsten.etherscan.io/tx/0x761221 aaaf916ec463175e9780effde11e9cde60b4d14cfb1f4f121d178d86b2 \\
\hline $100924.25 \mathrm{~Pa}$ & https $/ /$ ropsten.etherscan.io/tx/0xbecde93f9eadecede3d0edc837f34e81bf66e848a5ce82c23c4f0723a5549e96 \\
\hline
\end{tabular}

- Gambar 8. Data Pengujian Sensor Tekanan dan Hasil Validasi

\section{SUHU}

Sensor suhu banyak digunakan dalam berbagai peralatan, baik untuk keperluan industri mau pun pada peralatan elektro medis. Oleh karena itu, sensor suhu menjadi salah satu sensor yang digunakan dalam pengujian modul ini. Sensor suhu yang digunakan memiliki rentang untuk pengukuran suhu tubuh manusia. Data-data yang diuji menunjukkan sensitivitas sensor dan fleksibilitas pengiriman data ke EVM. Gambar 9 merupakan gambar yang memperlihatkan contoh keberhasilan pengiriman data ke Ropsten. Detail pengiriman terlihat dengan jelas pada Gambar 9. Di Gambar 10, diperlihatkan 20 data yang dikirimkan untuk pengujian modul. 
[ This is a Ropsten Testnet Transaction Only ]

\begin{tabular}{|c|c|}
\hline Transaction Hash: & 0x0cfb27de1b835c6e4e63c1b4aa46093216ae85cf06ff929e066a94c7193a88d1 [5 \\
\hline Status: & - success \\
\hline Block: & 536490622 Block Confirmations \\
\hline TimeStamp: & (1) 6 mins ago (Apr-08-2019 01:09:52 PM +UTC) \\
\hline From: & 0x9ad8ee5e185455d6e7205bf63ce3808eda44a2ff [t] \\
\hline To: & $0 \times 81 b 7 e 08 f 65 b d f 5648606 c 89998 a 9 c c 8164397647$ th \\
\hline Value: & 34 wei $(\$ 0.00)$ \\
\hline Transaction Fee: & 0.00037800000035 Ether $(\$ 0.000000)$ \\
\hline Gas Limit: & 210,000 \\
\hline Gas Used by Transaction: & $21,000(10 \%)$ \\
\hline Gas Price: & 0.000000018000000017 Ether $(18.000000017$ Gwei) \\
\hline
\end{tabular}

- Gambar 9. Bukti Keberhasilan Pengiriman Data Sensor Suhu

\begin{tabular}{|c|c|}
\hline Value & Etherscan \\
\hline $29.125^{\circ} \mathrm{C}$ & https://ropsten.etherscan.io/tx/0x5e542a63b0c300538f301da24ad8073f34de6ef0b4683ea0f050914d94bab809 \\
\hline $29^{\circ} \mathrm{C}$ & https://ropsten.etherscan.io/tx/0xc9a 4ae $4 \mathrm{c} 372 \mathrm{fa} 20 \mathrm{~d} 8 \mathrm{cc} 7253 \mathrm{e} 6 \mathrm{~d} 4 \mathrm{c} 15 \mathrm{~b} 1 \mathrm{e} 2 \mathrm{badcc} 5 \mathrm{bd} 0612 \mathrm{c} 386 \mathrm{c} 3 \mathrm{e} 38 \mathrm{~d} 8228 \mathrm{e} 1 \mathrm{e} 1$ \\
\hline $29.0625^{\circ} \mathrm{C}$ & https $/ /$ ropsten etherscan.io/tx/0xfbdaalfc3fc $7 b 3 \mathrm{~b} 014 \mathrm{c} 5 \mathrm{~d} 8 \mathrm{ace} 979 \mathrm{c} 5 \mathrm{f} 238 \mathrm{f} 0628 \mathrm{~b} 1 \mathrm{aae} 4806 \mathrm{~b} 8830 \mathrm{cc} 245 \mathrm{c} 1 \mathrm{fb} 18$ \\
\hline $29.0625^{\circ} \mathrm{C}$ & https://ropsten. etherscan.io/tx/0x7fb373df8d319424040a7118d74ee93eab3d6a3fdb3adfd5d58d764c0c4da09f \\
\hline $29.0625^{\circ} \mathrm{C}$ & https:/ropsten etherscan.io/tx/0xf37b06eeebd68696ebd3acb7f6dbe05b9a787a3ede36b0cf0c8c3b52d2928877 \\
\hline $29.1875^{\circ} \mathrm{C}$ & https://ropsten.etherscan.io/tx/0xbfdcace94bab8f003adlfcc9dc8d70abf8ba36bf95af6cf161254f310937fdc1 \\
\hline $29.0625^{\circ} \mathrm{C}$ & https://ropsten.etherscan.io/tx/0x $73 \mathrm{e} 41$ ee $4921 \mathrm{fc} 6 \mathrm{~b} 28543864252 \mathrm{bbd} 8688 \mathrm{a} 2227 \mathrm{~d} 1 \mathrm{fa} 0 \mathrm{c} 25857448735 \mathrm{cbaf} 312 \mathrm{~d} 4$ \\
\hline $29.125^{\circ} \mathrm{C}$ & https://ropsten etherscan.io/tx/0xfle7aebe4bb3e187740620bb35c731631ed7dad64d2aa6bc16fba32d2f2df870 \\
\hline $29.125^{\circ} \mathrm{C}$ & https $/ /$ ropsten etherscan.io/tx/0xfe3a53b173c0d314ceb744785631c22b5a33490667666f26485515c4c5dcaa56 \\
\hline $29.0625^{\circ} \mathrm{C}$ & https://ropsten.etherscan.io/tx/0xf75b497ff458c9d143af22c76ab92499d2a9e35b294c3b4b2ce21fbf9d010817 \\
\hline $29^{\circ} \mathrm{C}$ & https:/ropsten etherscan.io/tx/0x841aa81c0484416d058898419719f890cba3e6daf86fa748419288f7200a99a2 \\
\hline $29.0625^{\circ} \mathrm{C}$ & https $/ /$ ropsten etherscan.io/tx/0xbcfb58c731bd1c35b9211e98a00fb2fab0b5d96607c05d314a993e069d10dc72 \\
\hline $31.4375^{\circ} \mathrm{C}$ & https://ropsten etherscan.io/tx/0x14e711c84a2fc504fdb26cc92c6d21af55a957f953b8c0d5e073d9da76b6925a \\
\hline $37.875^{\circ} \mathrm{C}$ & https://ropsten etherscan.io/tx/0x2d0b1355a636dcb97680ff98511c3bd4dfa255c8f48735ce5309986635568925 \\
\hline $34.375^{\circ} \mathrm{C}$ & https://ropsten.etherscan.io/tx/0x6d7c32fe046541b43d944a12c103c0d88f8b8fce70a98cb6eef6a391629bf163 \\
\hline $31.9375^{\circ} \mathrm{C}$ & https://ropsten etherscan.io/tx/0x59c95de02d783f8flb3f37f529cebe768da4760c0d98dfd8094844d9d154ed46 \\
\hline $30.125^{\circ} \mathrm{C}$ & https $/ /$ ropsten etherscan.io/tx/0x0c71793e7e5d0453flda1a63249fdf1211a1822flca2621c7b1208325bfeffe 8 \\
\hline $29.75^{\circ} \mathrm{C}$ & https:/ropsten etherscan.io/tx/0x3df7aaa9af9de3b1401dlf64822493969387388c5b104e3a14dd99335465f4914 \\
\hline $38.0625^{\circ} \mathrm{C}$ & https://ropsten.etherscan.io/tx/0x2259115788dfc861c1c56d44c6ab2f8e0564bdc18331510b9fe2b1fe6613415b \\
\hline $39.875^{\circ} \mathrm{C}$ & https://ropsten.etherscan.io/tx/0x0826ebe162d8f1650a89b2176eefbd898f4239ee4ffd72bd7b8491d0dec41683 \\
\hline
\end{tabular}

- Gambar 10. Data Pengujian Sensor Suhu dan Hasil Validasi

\section{KESIMPULAN}

Kesimpulan yang dapat diambil dari perancangan ini adalah sebagai berikut:

1. Proses signing berhasil dilakukan dan dapat divalidasi dengan etherscan.io. Ini berarti blockchain dapat digunakan untuk melakukan sekuritisasi data IoT.

2. Proses ini supaya berhasil memerlukan delay pengiriman data dengan nilai minimum tertentu. Dalam pengujian ini, didapatkan bahwa delay 30 detik adalah nilai minimum agar data dapat terekam dengan baik di EVM.

\section{DAFTAR PUSTAKA}

[1] S. Smith, Internet of Things connected devices to triple by 2021, reaching over 46 billion units, in Juniper Research, 2016. 
[2] K. Rawlinson. Hp study reveals 70 percent of internet of things devices vulnerable to attack. [Online]. Available: http://www8.hp.com/us/en/ hp-news/press-release.html?id=1744676\#.WUrrwWgrKM8, diakses pada tanggal 29 Agustus 2018 pada jam 21.00 WIB.

[3] K. Christidis and M. Devetsikiotis, Blockchains and Smart Contracts for the Internet of Things, IEEE Access, vol. 4, 2016, pp. $2292-2303$.

[4] P. Danzi, et. al., Analysis of the Communication Traffic for Blockchain Synchronization of IoT Devices, IEEE International Conference on Communications (ICC), 2018.

[5] J. Pan and E. Alqrem, EdgeChain: An Edge-IoT Framework and Prototype Based on Blockchain and Smart Contracts, https://arxiv.org/abs/1806.06185, 2018, diakses pada tanggal 29 Agustus 2018 pada jam 22.00 WIB.

[6] U. Guin, P. Cui and A. Skjellum, Ensuring Proof-of-Authenticity of IoT Edge Devices using Blockchain Technology, The 2018 IEEE International Conference on Blockchain, 2018.

[7] A. Dorri, et. al., Blockchain for IoT Security and Privacy: The Case Study of a Smart Home, IEEE Percom Workshop on Security Privacy and Trust in The Internet of Thing, 2017.

[8] S. F. T. O. Mendonca, J. F. S. Junior and F. M. R. Alencar, The Blockchain-based Internet of Things Development: Initiatives and Challenges, ICSEA, 2017, pp. 28-33.

[9] J. Kogure, et. al., The Blockchain-based Internet of Things Development: Initiatives and Challenges, Fujitsu Scientific \& Technical Journal, vol. 53, no. 5, pp 56-61, 2017.

[10] A. Dorri, et. al., LSB: A Lightweight Scalable BlockChain for IoT Security and Privacy, https://arxiv.org/abs/1712.02969, 2017, diakses pada tanggal 20 September 2018 pada jam 23.00 WIB.

[11] A. Narayanan et al., Bitcoin and Cryptocurrency Technologies: A Comprehensive Introduction, Princeton University Press, 2016. 